ORIGINAL

\title{
Microdureza del esmalte dental en incisivos centrales permanentes de dos genotipos bovinos
}

\section{Dental enamel micro-hardness for permanent central incisors in two beef cattle genotypes}

\author{
Eduardo Fernández T, ${ }^{1 *}$ Esp, Nidia Abbiati $C_{1}{ }^{2}$ M.Sc, Jorge Cabrera A, ${ }^{3}$ Ing, \\ Rubén Martínez M, ${ }^{1}$ Ph.D.
}

\begin{abstract}
1Universidad Nacional de Lomas de Zamora, Facultad de Ciencias Agrarias, Genética Animal, Camino de Cintura km 2, Llavallol (1836), Argentina. ${ }^{2}$ Universidad Nacional de Lomas de Zamora, Facultad de Ciencias Agrarias, Estadística y Diseño Experimental, Camino de Cintura km 2, Llavallol (1836), Argentina. ${ }^{3}$ Universidad Nacional de Lomas de Zamora, Facultad de Ingeniería, Laboratorio de Materiales, Complejo Universitario, Juan XXIII y Ruta Provincial 4, Lomas de Zamora (1832), Argentina. *Correspondencia: ednfer@yahoo.com
\end{abstract}

Recibido: Diciembre de 2009; Aceptado: Julio 2010.

\section{RESUMEN}

Objetivo. Evaluar la microdureza del esmalte dental de novillos criollos de origen patagónico y cruza índica criados en ambientes diferentes. Materiales y métodos. Se utilizaron dientes incisivos centrales derechos de novillos adultos: ocho Criollos Patagónicos (GCP) y siete Cruzas Índicas (GCI). Para cada diente se definió una transecta central recorriendo el diente desde el borde externo del esmalte hacia la dentina y se midió la dureza en tres profundidades equidistantes: a $30 \mu$ del borde exterior (P1), a $30 \mu$ previos a la conexión amelodentinaria (P3) y P2 equidistante entre P1 y P3. Las mediciones de microdureza se realizaron con un microdurómetro Shimadzu modelo HMV-2 y se expresaron en unidades Vickers. Para el análisis se utilizó un modelo de regresión aleatoria. Se empleó el criterio de información de Akaike para la elección de la estructura de las matrices asociadas a los efectos aleatorios. Para el análisis estadístico se empleó el procedimiento PROC MIXED de SAS, y un nivel de significación del $5 \%$. Resultados. No se detectó falta de paralelismo entre los grupos ( $p>0.05)$, con lo cual se ajustaron rectas de igual pendiente en cada grupo. La dureza media del esmalte disminuyó al aumentar la profundidad de la medición para ambos genotipos. Se observó heterogeneidad en la variabilidad de los grupos. Conclusiones. El GCP presentó mayor dureza pero fue el de mayor variabilidad. Se confirma la disminución de la dureza media con el aumento de la profundidad en el esmalte independientemente del genotipo y el ambiente.

Palabras clave: Evaluación, esmalte dental, bovinos. (Fuente: DeCS) 
Fernández - Microdureza del esmalte dental de dos genotipos bovinos

\section{ABSTRACT}

Objective. To evaluate the microhardness of tooth enamel from creole steers of Patagonian origin and from indicus cross raised in different environments. Materials and methods. Right central incisors from adult steers were used: eight Patagonian Creole (PCG) and seven indicus cross (ICG). For each tooth was defined a central transect crossing the tooth from the outer edge of the enamel into the dentin, and hardness was measured at three equidistant depths: $30 \mu$ from the outer edge $(P 1), 30 \mu$ prior to the dentin-enamel junction (P3), and P2 between $\mathrm{P} 1$ and P3. Microhardness measurements were performed with a Shimadzu microdurometer model HMV-2 and were expressed in Vickers units. Data were analysed using a random regression model. Akaike information criterion was used for the election of the structure of the matrix associated to random effects. For the statistical analysis was used SAS PROC MIXED procedure, at a $5 \%$ significance level. Results. No lack of parallelism was detected between groups $(p>0.05)$, and lines of equal slope were adjusted in each group. The average hardness of enamel decreased with increasing depth of measurement for both genotypes. Heterogeneity was observed in the variability of the groups. Conclusions. Not only PCG showed more hardness but also had the highest variability. Decrease in average hardness with increasing depth in enamel regardless of genotype and environment was confirmed.

Key words: Evaluation, dental enamel, cattle. (Source: DeCS)

\section{INTRODUCCIÓN}

Desde el punto de vista anatómico un elemento dentario consta de una corona, un cuello y una raíz. La corona clínica es la porción libre del diente y la raíz la parte que se inserta en el hueso alveolar, fijándose al mismo por el ligamento periodontal. Pese a la gran variabilidad de los dientes en forma y tamaño, a nivel histológico tienen estructuras similares. Están formados por la dentina, el esmalte (que recubre a la dentina en la zona coronal), el cemento (que rodea a la dentina en la zona radicular) y por la cavidad pulpar (espacio ubicado dentro de la dentina) que contiene a la pulpa dentaria (tejido blando muy vascularizado e inervado) $(1,2)$.

La dentina es un tejido mineralizado (70\% de materia inorgánica) y metabólicamente activo. Esta última característica la diferencia del esmalte ya que le permite regenerarse luego de sufrir roturas. El esmalte dental es el tejido más duro de los mamíferos y se encuentra altamente mineralizado (95\% de materia inorgánica). Está constituido por cristales de hidroxiapatita que se organizan formando prismas de dirección y longitud variable. Contiene además un 1 a $2 \%$ de matriz orgánica y un 3 a $5 \%$ de agua $(1,2)$. Las piezas dentales bovinas constituyen componentes anatómicos que condicionan la vida útil de estos animales debido a que sufren un proceso natural de desgaste, de manera mucho más notable y determinante en los dientes incisivos. Si bien este proceso se relaciona fuertemente con las condiciones medio ambientales en las cuales viven los animales, en especial con el tipo de alimentación y la calidad del agua de bebida, existen antecedentes que muestran diferencias entre razas (3).

Nuñez-Dominguez et al (3) encontraron que para un mismo ambiente, animales de cruza británica presentaron ventajas en cuanto al tamaño y condición de los incisivos cuando se compararon con las razas puras que los originaron. Por otra parte Riley et al (4) propusieron para vacas de 14 años una clasificación en relación a la ausencia/ presencia de incisivos (mouth score), y concluyeron que, utilizando madres Hereford, el score medio era menor en hijas de padres Aberdeen Angus, que en hijas de padres de otras cinco razas destacadas. Algunos autores han destacado que el deterioro y desgaste temprano de los incisivos deciduos en la raza Hereford, en comparación con 
otra raza nativa en Zimbabwe, se debió a variaciones en la oclusión y en la dureza del esmalte $(5,6)$. La susceptibilidad a la abrasión aumenta cuando la dureza del esmalte disminuye (7). La dureza es una propiedad física definida como la capacidad que tiene la superficie de una determinada sustancia para resistir la penetración de una punta bajo determinada carga, por lo cual se constituye en un indicador de la calidad del esmalte dental para resistir procesos de desgaste (5). Algunos autores caracterizaron la microdureza del esmalte recorriendo distintas zonas a lo ancho de dientes incisivos bovinos, no encontrando diferencias significativas $(5,8)$. Se ha observado que vacas criollas argentinas de origen patagónico, criadas en un mismo ambiente, presentan una importante variabilidad en cuanto a su condición de desgaste dental. Se han descartado por dicha condición algunos vientres de doce años de edad y otros recién a los veinte años, presentando en ambos casos similar estado dental (9).

El objetivo de este trabajo fue estudiar la microdureza del esmalte dental a distintas profundidades desde el borde exterior en dirección a la conexión amelo-dentinaria en incisivos permanentes de dos grupos de animales de distinto genotipo y criados en ambientes diferentes.

\section{MATERIALES Y MÉTODOS}

Sitio de estudio, muestras, animales y procedimientos. Se trabajo con los dientes incisivos centrales derechos de dos grupos de novillos (3 a 3,5 años de edad), definidos según su genotipo y lugar de procedencia: el grupo de Criollos Patagónicos provenientes de la provincia de Buenos Aires (GCP) y el grupo, Cruza Índica oriundos del Departamento San Cristóbal, provincia de Santa Fe (GCI) (Figura 1). Se utilizaron ocho piezas dentales de GCP y siete de GCI. En cada pieza se efectuó un corte transversal a la altura media de la corona empleando un torno manual con cuchilla de diamante. La parte superior del diente se incluyó en baquelita (Figura 2), para luego ser pulida y lograr una superficie

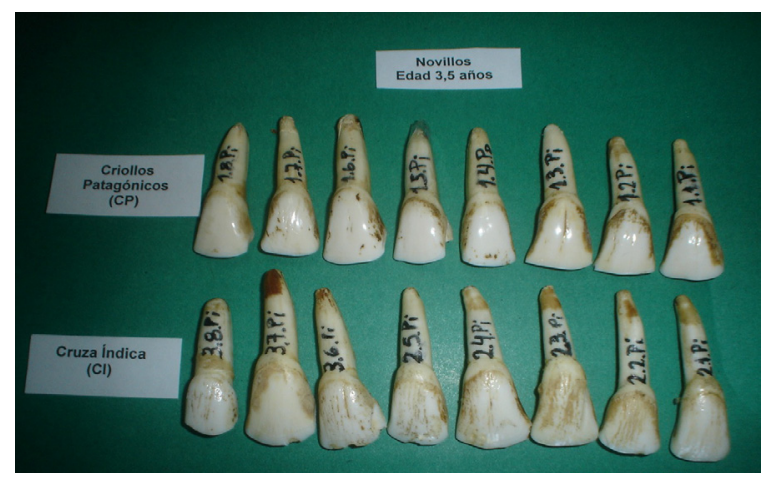

Figura 1. Piezas dentales de ambos grupos.

más pareja que facilitó la observación. Se definió una transecta central recorriendo el diente desde el borde externo del esmalte hacia la dentina y se midió la dureza en tres profundidades, dependientes del espesor del esmalte, equidistantes: a $30 \mu$ del borde exterior (P1), a $30 \mu$ previos a la conexión amelodentinaria (P3) y P2 entre P1 y P3 (Figura 3). La medición de la microdureza se expresó en unidades Vickers, empleándose a tal fin un microdurómetro con microscopio incorporado, marca (SHIMADZU, Kyoto, Japan) Modelo HMV-2 (Figura 4), aplicando una carga de $100 \mathrm{~g}$. Este instrumento cuenta con un diamante en forma de pirámide de base cuadrada, con ángulo entre las caras de $136^{\circ}$. El cálculo del valor de dureza Vickers, se obtuvo automáticamente luego de medir la longitud de las diagonales de la impronta obtenida sobre la superficie del esmalte.

Análisis estadístico. Se empleó un modelo de regresión aleatoria donde la parte fija relacionó la microdureza media con la profundidad a través de una ecuación lineal para cada grupo.

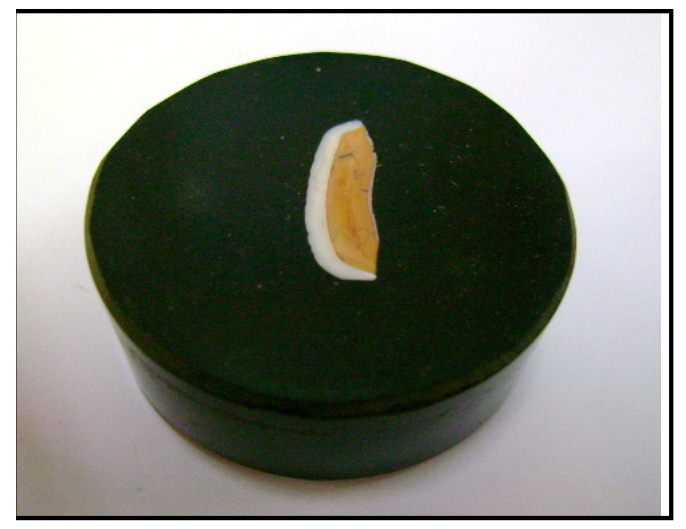

Figura 2. Inclusión del corte en baquelita. 


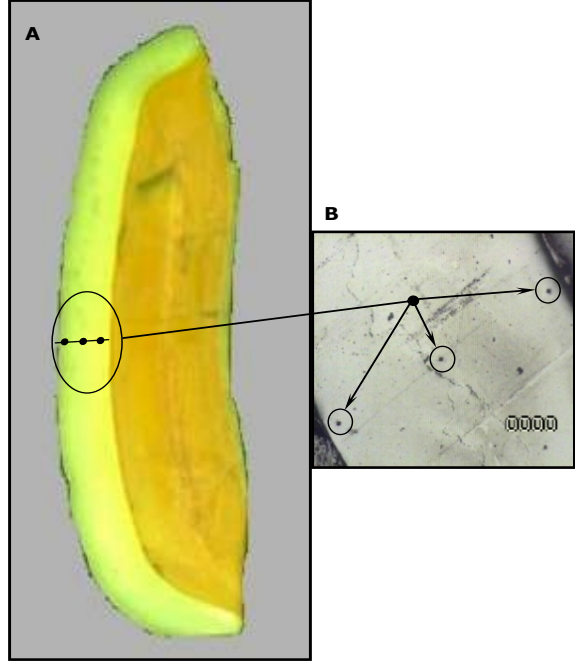

Figura 3. A: Corte transversal de diente incisivo central donde se observa el esmalte (parte externa) y la dentina (interna) y transecta sobre la que se realizaron las mediciones. B: P1, P2 y P3, improntas que realiza el identador, mediante las cuales se determina la dureza del material.

La parte aleatoria expresa la misma relación lineal en cada pieza dental como desvío de la recta media del grupo al que pertenece; para los coeficientes aleatorios se consideraron matrices de varianzas y covarianzas heterogéneas, según grupo. Se empleó el criterio de información de Akaike para la elección de la estructura de las matrices de varianzas y covarianzas asociadas a los efectos aleatorios. Se utilizó el procedimiento PROC MIXED, con ajuste de los grados de libertad mediante el método "containment", que consiste en calcular los grados de libertad del denominador de cada efecto fijo como el número total de observaciones menos el mínimo de los rangos de la matriz ampliada conformada por las matrices de incidencia de los efectos fijos y aleatorios que contienen al fijo. Se trabajó con un nivel de significación del $5 \%$. El procesamiento de los datos se realizó por medio del software SAS (10).

\section{RESULTADOS}

La tabla 1 muestra el valor promedio y el desvío estándar de microdureza de cada pieza dental en ambos grupos y la

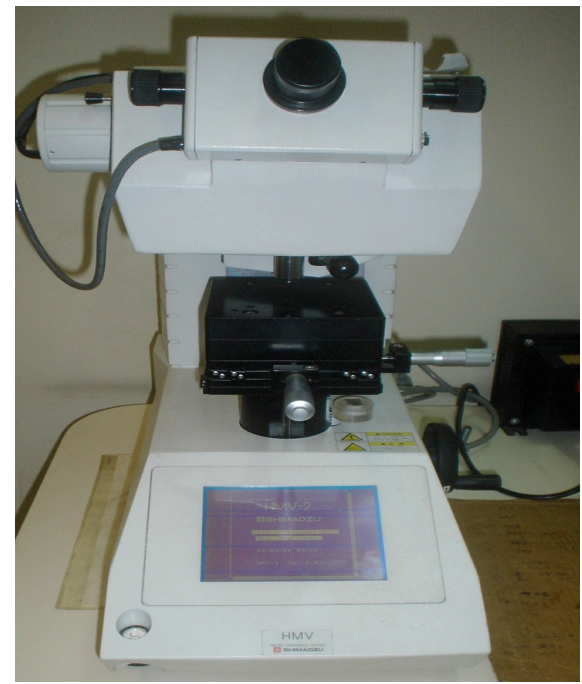

Figura 4. Microdurómetro (SHIMADZU, Kyoto, Japan) Modelo HMV-2.

tabla 2 informa los mismos estadísticos para cada grupo y cada profundidad.

Tabla 1. Unidades Vickers (IDE) en cada incisivo según grupo.

\begin{tabular}{ccccccccc}
\hline \multirow{2}{*}{ Grupo } & \multicolumn{7}{c}{ Incisivos } \\
\cline { 2 - 8 } & 1 & 2 & 3 & 4 & 5 & 6 & 7 & 8 \\
\hline \multirow{2}{*}{ GCP } & 369.33 & 207.33 & 289.67 & 444.33 & 370.67 & 425.33 & 428.33 & 417.67 \\
& $(103.37)$ & $(72.39)$ & $(122.54)$ & $(42.06)$ & $(117.50)$ & $(95.85)$ & $(120.14)$ & $(83.79)$ \\
\multirow{2}{*}{ GCI } & 387.00 & 296.00 & 302.00 & 295.33 & 337.67 & 306.67 & 334.67 & \\
& $(46.68)$ & $(71.92)$ & $(59.92)$ & $(67.01)$ & $(79.00)$ & $(92.09)$ & $(96.44)$ & \\
\hline
\end{tabular}

$\mathrm{GCP}=$ Grupo Criollo Patagónico, GCI= Grupo Cruza Índica

Tabla 2. Unidades Vickers (IDE) según profundidad y grupo.

\begin{tabular}{ccc}
\hline Profundidad & \multicolumn{2}{c}{ Grupo } \\
\cline { 2 - 3 } & GCP & GCI \\
\hline P1 & 445.25 & 390.71 \\
& $(81.42)$ & $(37.51)$ \\
P2 & 395.38 & 321.86 \\
& $(92.98)$ & $(51.11)$ \\
P3 & 266.63 & 255.71 \\
& $(87.01)$ & $(42.71)$ \\
Media & 369.08 & 322.76 \\
D.E. & $(113.41)$ & $(70.30)$ \\
\hline
\end{tabular}

$\mathrm{GCP}=$ Grupo Criollo Patagónico, GCI=Grupo Cruza Índica, P1 a P3=profundidades externa, media e interior del esmalte, respectivamente. 
Una matriz de simetría compuesta, diferente para cada raza, resultó adecuada para modelar las matrices de varianzas de los efectos aleatorios (co). El estudio de residuos no detectó desvíos de los supuestos. En la tabla 3 se presenta el análisis de varianza parcial para los efectos fijos.

Tabla 3. Análisis de varianza para los efectos fijos.

\begin{tabular}{|c|c|c|c|c|}
\hline Efecto & $\begin{array}{c}\text { G.L } \\
\text { Numerador }\end{array}$ & $\begin{array}{c}\text { G.L } \\
\text { Denominador }\end{array}$ & $F$ & $\mathrm{Pr}>\mathrm{F}$ \\
\hline Profundidad & 1 & 13 & 67.92 & $<.0001$ \\
\hline Grupo & 1 & 13 & 4.87 & 0.0459 \\
\hline $\begin{array}{l}\text { Profundidad } \\
{ }^{*} \text { Grupo }\end{array}$ & 1 & 13 & 2.82 & 0.1167 \\
\hline
\end{tabular}

No se detectó falta de paralelismo entre los grupos $(p>0.05)$, con lo cual se ajustaron rectas de igual pendiente en cada grupo (Tabla 4 ).

Tabla 4. Estimación de los efectos fijos

\begin{tabular}{lcc}
\hline \multicolumn{1}{c}{ Parámetros } & Estimación & Error Estándar \\
\hline Ordenada al Origen GCP & 473.91 & 24.72 \\
Ordenada al Origen GCI & 404.88 & 13.82 \\
Pendiente & -125.21 & 15.23 \\
\hline
\end{tabular}

En las figuras 5 y 6 (uno por cada grupo) se muestra las regresiones aleatorias de cada diente y las rectas estimadas. Puede observarse la heterogeneidad en la variabilidad de los grupos.

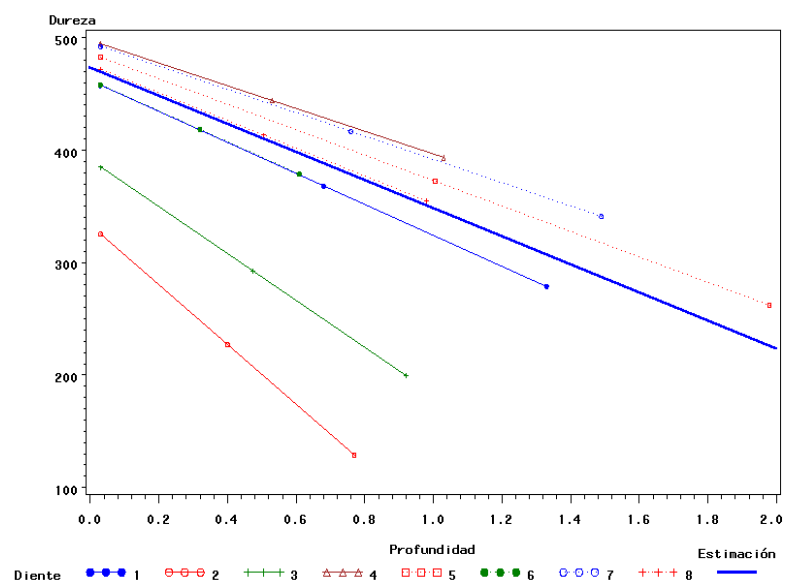

Figura 5. Regresiones aleatorias y recta estimada para GCP.

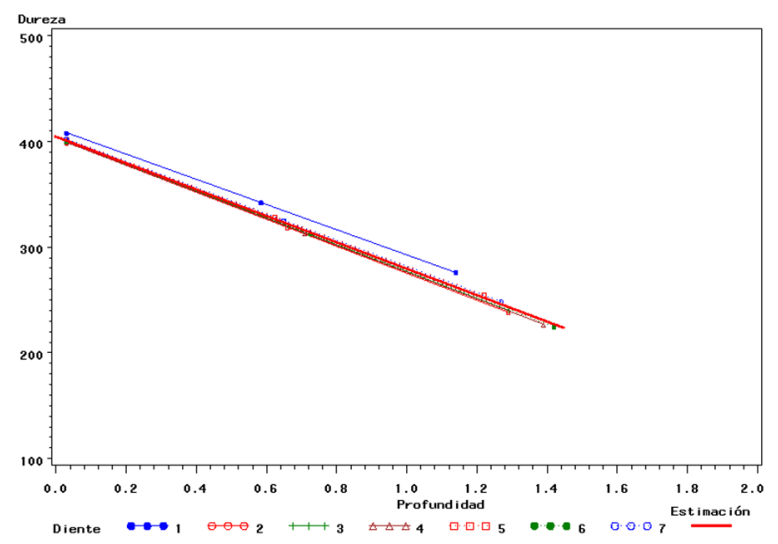

Figura 6. Regresiones aleatorias y recta estimada para GCI.

\section{DISCUSIÓN}

No se evidenció interacción entre los gruposy la profundidad, presentándose un gradiente negativo desde el borde del esmalte en dirección a la dentina. Este último resultado concuerda con los presentados por otros autores $(11,12,8)$. Este hecho podría estar asociado al gradiente de carbonato hallado por Zazzo et al (13) que provoca, según estos autores, propiedades cristalográficas diferentes en la apatita del esmalte externo e interno. Esta disminución en la dureza en dirección a la conexión amelodentinaria (CAD) podría resultar razonable debido a que la dentina presenta un valor de dureza muy inferior al que tiene el esmalte (14). Marshall et al (15) observaron, empleando técnicas de nanoidentación, que $10 \mu$ antes de la CAD se produce una caída abrupta de la nanodureza, hecho que favorece la integridad del diente.

El GCP presentó mayor dureza que GCI, pero fue el de mayor variabilidad. Existen evidencias de que la dureza de dientes permanentes de razas adaptadas a condiciones ambientales no favorables, es mayor que en otras razas exóticas (5). En tal sentido la microdureza superior en el grupo GCP (criado en la provincia de Buenos Aires) podría deberse a los muchos años de adaptación a un medio ambiente muy hostil como es el del Parque Nacional Los Glaciares en la cordillera patagónica argentina. 
En conclusión, el grupo criollo patogenico presentó mayor dureza pero igualmente mayor variabilidad. Se confirma la disminución de la dureza media con el aumento de la profundidad en el esmalte independientemente del genotipo y el ambiente.

\section{Agradecimientos}

Expresamos nuestro agradecimiento al Prof. Silvio Zalcman del Laboratorio de Materiales de la Facultad de Ingeniería de la UNLZ por su desinteresada atención y colaboración en la preparación de las muestras.

\section{REFERENCIAS}

1. Ferraris Gómez ME, Muñoz Campos A. Histología y embriología bucodental. 2da. Edición. Madrid: Editorial Médica Panamericana; 2006.

2. Frandson RD y Whitten EH. Anatomía y Fisiología de los Animales Domésticos 3ra Edición. México: Editorial Interamericana; 1984.

3. Nunez-Dominguez $R$, Cundiff LV, Dickerson GE, Gregory KE, Koch RM. Heterosis for survival and dentition in Hereford, Angus, Shorthorn, and crossbred cows . J Anim Sci 1991; 69:1885-1898.

4. Riley JO, Sanders DG, Knutson RE, Lunt DK. Comparison of F1 Bos indicus $x$ Hereford cows in central Texas: II. Udder, mouth, longevity, and lifetime productivity. J Anim Sci 2001; 79(6):1439-1449.

5. Steenkamp JDG. Effect of brittle hardness and abrasive hardness of enamel on degree of attrition of deciduous teeth of representative breeds of Bos indicus and Bos taurus origin. Agroanimalia 1969; 1:23-34.

6. Steenkamp JDG. Differences in manner of occlusion of representative indigenous and exotic breeds of cattle and effect on wear of deciduous incisor teeth. Agroanimalia 1970; 2:85-92.
7. Attin T, Koidl U, Buchalla W, Schaller HG, Kielbassa AM, Hellwig E. Correlation of microhardness and wear in differently eroded bovine dental enamel. Arch Oral Biol 1997; 42(3):243-50.

8. Fernández EN, Abbiati NN, Abal A, Durso G, Cabrera J, Martínez RD. Análisis exploratorio de la dureza del esmalte en piezas dentales bovinas empleando modelos mixtos. XII Reunión del Grupo Argentino de Biometría GAB San Miguel de Tucumán, Provincia de Tucumán, Argentina; 2008.

9. Martínez R. Caracterización Genética y Morfológica del bovino criollo de origen patagónico. [Tesis doctoral]. España: Universidad Politécnica de Valencia, Departamento de Ciencia Animal. 2008.

10. SAS 9.2. SAS Institute Inc. 2009.

11. Fong $H$, Sarikaya $M$, White $S N$, Snead ML. Nano-mechanical properties profiles across dentin-enamel junction of human incisor teeth. Mater Sci Eng 2000; C7:119-128.

12. Angker $L$, Swain MV, Kilpatrick N. Micro-mechanical characterisation of the properties of primary tooth dentine Journal of Dentistry 2003. 31(4):261. 
13. Zazzo A, Balasse M, Patterson WP. High-resolution $\delta 13 \mathrm{C}$ intratooth profiles in bovine enamel: Implications for mineralization pattern and isotopic attenuation. Geochim Cosmochim Acta 2005; 69(14):3631-3642.

14. Fernández EN, Abbiati NN, Abal A, Abad $\mathrm{R}$, Martinez RD. Análisis exploratorio para el perfil de dureza en piezas dentales bovinas empleando modelos mixtos. VIII Congreso Latinoamericano de Sociedades de Estadística: Montevideo, Uruguay; 2008.
15. Marshall GW, Balooch M, Gallagher RR, Gansky SA, Marshall SJ. Mechanical properties of the dentinoenamel junction: AFM studies of nanohardness, elastic modulus, and fracture. J Biomed Mater Res 2001; 54:87-95. 